\title{
Experience with FreeStyle Libre Flash glucose monitoring system in management of refractory dumping syndrome in pregnancy shortly after bariatric surgery
}

\author{
Peter Novodvorsky1,2, Emma Walkinshaw1,2, Waliur Rahman', Valerie Gordon', \\ Karen Towse', Sarah Mitchell', Dinesh Selvarajah',2, Priya Madhuvrata ${ }^{3}$ and AliaMunir ${ }^{1,2}$ \\ 1Department of Diabetes and Endocrinology, Sheffield Teaching Hospitals NHS Foundation Trust, Sheffield, UK, \\ 2Department of Oncology and Metabolism, University of Sheffield, Sheffield, UK, and ${ }^{3}$ Department of Obstetrics, \\ Gynaecology and Neonatology, Sheffield Teaching Hospitals NHS Foundation Trust, Sheffield, UK
}

\author{
Correspondence \\ should be addressed \\ to P Novodvorsky \\ Email \\ p.novodvorsky@sheffield. \\ ac.uk
}

\section{Summary}

Bariatric surgery is an effective therapy for obesity but is associated with long-term complications such as dumping syndromes and nutritional deficiencies. We report a case of a 26-year-old caucasian female, with history of morbid obesity and gestational diabetes (GDM), who became pregnant 4 months after Roux-en-Y bypass surgery. She developed GDM during subsequent pregnancy, which was initially managed with metformin and insulin. Nocturnal hypoglycaemia causing sleep disturbance and daytime somnolence occured at 19 weeks of pregnancy (19/40). Treatment with rapid-acting carbohydrates precipitated further hypoglycaemia. Laboratory investigations confirmed hypoglycaemia at $2.2 \mathrm{mmol} / \mathrm{L}$ with appropriately low insulin and C-peptide, intact HPA axis and negative IgG insulin antibodies. The patient was seen regularly by the bariatric dietetic team but concerns about compliance persisted. A FreeStyle Libre system was used from $21 / 40$ enabling the patient a real-time feedback of changes in interstitial glucose following high or low Gl index food intake. The patient declined a trial of acarbose but consented to an intraveneous dextrose infusion overnight resulting in improvement but not complete abolishment of nocturnal hypoglycaemia. Hypoglycaemias subsided at 34/40 and metformin and insulin had to be re-introduced due to high post-prandial blood glucose readings. An emergency C-section was indicated at $35+1 / 40$ and a small-for-gestational-age female was delivered. There have been no further episodes of hypoglycaemia following delivery. This case illustrates challenges in the management of pregnancy following bariatric surgery. To our knowledge, this is the first use of FreeStyle Libre in dumping syndrome in pregnancy following bariatric surgery with troublesome nocturnal hypoglycaemia.

\section{Learning points:}

- Bariatric surgery represents the most effective treatment modality in cases of severe obesity. With increasing prevalence of obesity, more people are likely to undergo bariatric procedures, many of which are women of childbearing age.

- Fertility generally improves after bariatric surgery due to weight reduction, but pregnancy is not recommended for at least 12-24 months after surgery. If pregnancy occurs, there are currently little evidence-based guidelines available on how to manage complications such as dumping syndromes or gestational diabetes (GDM) in women with history of bariatric surgery.

- Diagnosis of GDM relies on the use of a $75 \mathrm{~g}$ oral glucose tolerance test (OGTT). The use of this test in pregnant women is not recommended due to its potential to precipitate dumping syndrome. Capillary glucose monitoring profiles or continuous glucose monitoring (CGM) is being currently discussed as alternative testing modalities. 
- As the CGM technology becomes more available, including the recently introduced FreeStyle Libre Flash glucose monitoring system, more pregnant women, including those after bariatric surgery, will have access to this technology. We suggest urgent development of guidelines regarding the use of CGM and flash glucose monitoring tools in these circumstances and in the interim recommend careful consideration of their use on a case-to-case basis.

\section{Background}

Bariatric surgery represents a very effective method in the treatment of morbid obesity and related complications. Bariatric procedures induce major changes in the structure and function of the gastrointestinal tract leading to increased propensity to early and late dumping syndromes (DS) and nutritional deficiencies (1). The latest UK national bariatric surgery registry from 2014 reports that from 12869 women who had bariatric surgery between 2011 and 2013, nearly 70\% were of childbearing age (2), and these numbers are likely to rise since the prevalence of obesity increases. Fertility improves following bariatric surgery due to improved ovulation rates linked with weight loss; however, women are advised to avoid pregnancy for 12-24 months after bariatric intervention to reduce the risk of intrauterine growth retardation caused by the ongoing catabolic state (3). To our knowledge, data on pregnancy numbers after bariatric surgery in the UK are currently not available.

Pregnancy constitutes a state of increased insulin resistance. Gestational diabetes mellitus (GDM) is defined as diabetes diagnosed during the second or third trimester of pregnancy that was not clearly overt prior to gestation. It is linked with an increased risk of adverse outcomes for the mother and for the baby (4). A significant proportion of women who had bariatric intervention, despite having lost significant amount of weight, would still have a body mass index (BMI) $>30 \mathrm{~kg} / \mathrm{m}^{2}$ at the time of booking appointment or would have had previous history of GDM thus fulfilling the current National Institute for Health and Care Excellence (NICE) criteria for GDM screening (5). A standard screening and diagnostic test for GDM, the $75 \mathrm{~g}$ 2-h oral glucose tolerance test (OGTT) is considered to be risky in patients after bariatric surgery due to its potential to cause DS as a result of altered glycaemic responses. Alternative approaches such as capillary glucose monitoring or the use of continuous glucose monitoring (CGM) are currently being debated (6). In addition, once diagnosed, GDM in women after bariatric surgery constitutes a challenging clinical condition requiring multidisciplinary management with surgical, medical, obstetric and dietetic input with currently little evidence-based guidelines available (3).

FreeStyle Libre Flash glucose monitoring system (FL) (Abbott Diabetes Care, Maidenhead, UK) represents a variation of CGM that has been recently introduced into clinical practice. It consists of a sensor that measures interstitial glucose (IG) levels at closed spaced intervals with a storage capacity of $8 \mathrm{~h}$ of IG data and of a reader with a monitor. In order to get a coherent IG profile, the user is asked to swipe the reader over the sensor at least every $8 \mathrm{~h}$. IG data are displayed on the monitor of the reader together with IG trends. FL thus provides a useful and less costly alternative to standard CGM despite its recognised limitations such as inability to set alarms for high or low IG levels and a recognised tendency to lower-than-expected IG values in lower glucose ranges compared to the reference method (7).

\section{Case presentation}

A 26-year-old Caucasian female with history of morbid obesity (pre-surgery BMI $44.3 \mathrm{~kg} / \mathrm{m}^{2}$ ) and GDM in her two previous pregnancies underwent Roux-en-Y gastric bypass (RYGB) surgery and became pregnant four months later. The patient had a background of polycystic ovarian syndrome, endometriosis, laparoscopic right ovarian cystectomy, appendectomy and vitamin D deficiency. She also developed mild cholestasis of pregnancy and was treated with ursodeoxycholic acid. There was also a history of complex social circumstances and domestic abuse. There was no family history of obesity, diabetes, GDM or any other endocrine condition in her first-degree relatives. Her brother and grandfather from mother's side had a history of lung emphysema. She has received the standard dietary education and counselling provided by the bariatric dietician team for people undergoing bariatric interventions at Sheffield Teaching Hospitals NHS Foundation Trust before and subsequently after her bariatric surgery. This included provision of detailed verbal and written information regarding necessary 
dietary modifications, vitamin and micronutrient supplementation together with the recommendation of avoiding pregnancy for 12-24 months after the bariatric surgery. During the course of pregnancy, she has been taking vitamin and micronutrient supplementation (WLS Forte and Calcium Plus, FitForMe, Rotterdam, Netherlands) and in addition, a cholecalciferol $800 \mathrm{IU}$ tablet per day.

As expected, she continued to lose weight in the months following the bariatric surgery, which continued during the course of pregnancy (Fig. 1).

Due to the previous history of GDM and bariatric surgery, the patient was offered capillary blood glucose monitoring at her booking appointment at 11th week of gestation (11/40), instead of the usual 2-h $75 \mathrm{~g}$ OGTT test. The recorded blood glucose (BM) values were diagnostic of GDM (6) (Table 1A) and metformin was started at 15/40. BM values continued to be elevated on metformin (Table 1B), and insulin (Humulin I and Humalog (Eli Lilly)) was added 2 weeks later.

At 19/40, this lady was admitted under the care of the Obstetrics and Gynaecology team with persistent vomiting. A week prior to admission (18/40), she began to record $\mathrm{BM}$ readings in the hypoglycaemic range that were symptomatic, with a tremor and increased sweating. Metformin and insulin were stopped at 19/40, but the post-prandial and nocturnal hypoglycaemia persisted. She had no history of chronic liver disease, and her liver function tests were normal apart from a mild elevation of bile acids in the range of $20-37 \mu \mathrm{mol} / \mathrm{L}$ (reference range (RR): $0-14 \mu \mathrm{mol} / \mathrm{L})$ throughout the 3rd trimester of her pregnancy in keeping with the diagnosis of cholestasis of pregnancy. Her renal function was normal.

\section{Investigation}

Laboratory investigations showed a blood glucose value of $2.2 \mathrm{mmol} / \mathrm{L}$ with appropriately low insulin levels (14.8 pmol/L,RR: $17.8-173 \mathrm{pmol} / \mathrm{L})$ and C-peptide levels (113 pmol/L, RR: 298-2350 pmol/L), negative IgG insulin antibodies and 09:00h cortisol value of $658 \mathrm{nmol} / \mathrm{L}$, indicating an intact hypothalamic-pituitary-adrenal axis.

\section{Treatment}

Diagnosis of late DS, most likely in combination with an early dumping phase, was established, and this lady received appropriate dietary advice from the bariatric dietetic team. She was advised to take frequent smallportioned meals, with the exclusion of high glycaemic

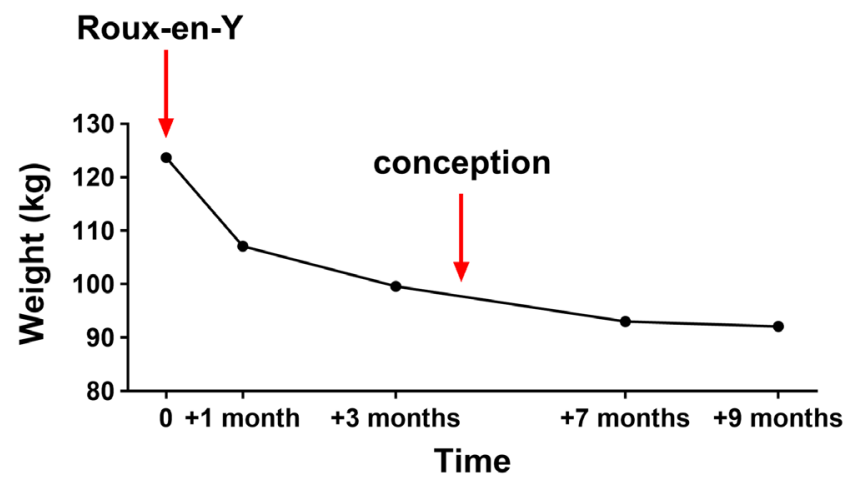

Figure 1

Weight chart of the 26-year-old lady with history of Roux-en-Y gastric bypass surgery who became pregnant shortly after. Times of bariatric intervention and approximate time of conception are indicated with arrows.

index (GI) food and a preference for low GI food. Avoidance of liquid carbohydrates and drinking fluids with meals $(1,3)$ was recommended. She found it difficult to adhere to dietary recommendations and treated hypoglycaemia with fast-acting carbohydrates resulting in subsequent hypoglycaemia and disturbed sleep with excess daytime tiredness. FL was introduced at 22/40 in order to avoid frequent nocturnal finger prick BM measurements and as an educational tool with the aim to help the patient to identify hypoglycaemic triggers (Fig. 2A).

Despite these measures, this lady continued to experience ongoing symptomatic hypoglycaemia that she was able to treat independently. Despite very low IG and BM readings (finger prick BM readings marked in Fig. 2 with *), there was no history of collapse, seizure or loss of consciousness. Disturbed sleep continued to have negative impact on her wellbeing. At 25/40, a decision was made to prevent nocturnal hypoglycaemia with continuous intravenous dextrose infusion overnight (00:00-07:00 h) (Fig. 2B). Poor compliance with dietary recommendations persisted despite ongoing support from

Table 1 Example of capillary blood glucose profiles on diet alone $(A)$ and on metformin (B).

\begin{tabular}{|c|c|c|c|c|c|}
\hline Fasting BM & $\begin{array}{c}1 \mathrm{~h} \\
\text { post }\end{array}$ & Pre-Iunch & $\begin{array}{c}1 \mathrm{~h} \\
\text { post }\end{array}$ & Pre-dinner & $\begin{array}{c}1 \mathrm{~h} \\
\text { post }\end{array}$ \\
\hline \multicolumn{6}{|c|}{ A. Diet alone } \\
\hline $6.3 *$ & 8.4 & 4.3 & 8.3 & 6.3 & 9.1 \\
\hline 5.4 & 8.4 & 5.4 & 9.5 & 6.5 & 10.1 \\
\hline \multicolumn{6}{|c|}{ B. On metformin } \\
\hline 6.0 & 7.2 & 7.0 & 9.3 & 6.3 & 8.3 \\
\hline 4.2 & 6.3 & 5.0 & 14.3 & 8.0 & 10.2 \\
\hline
\end{tabular}

Figures in bold are indicative of gestational diabetes mellitus (GDM).

*All figures are reported in $\mathrm{mmol} / \mathrm{L}$. 
the diabetes specialist nurses and bariatric dieticians. The patient declined a trial of acarbose due to concerns about potential risks to the unborn baby and was resistant to the suggestion of discharge with community support. This was substantiated by the fear of inability to treat her hypoglycaemia when on her own and not being able to look after her 2 young children. Gradually, intravenous dextrose infusions were discontinued (Fig. 2C), and the patient was discharged home at 29/40.

Following discharge, this lady did not attend an appointment with the diabetes team and she returned her FL device shortly after. She was reviewed in the antenatal clinic in later stages of her pregnancy (32/40), and because of elevated post-prandial BM values metformin (32/40) and later insulin (Humalog) were re-introduced (33/40). Hypoglycaemias were less prominent and resolved completely at 34/40. The estimated fetal weight (EFW), which was on the 50th centile during the earlier stages of pregnancy declined at later stages resulting in EFW at 5th centile at 33/40 (Fig. 3). Head circumference (HC) and abdominal circumference (AC) followed a similar trend.

A
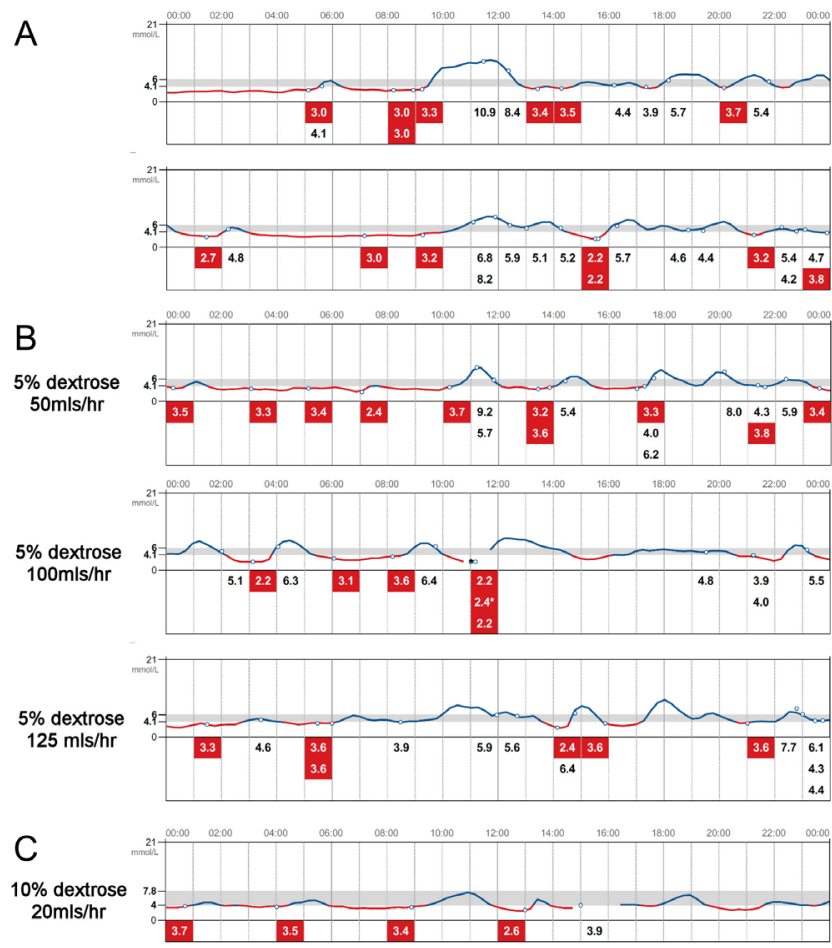

Figure 2

Interstitial glucose (IG) profiles uploaded from the FreeStyle Libre Flash glucose monitoring system. (A) IG profiles with dietary interventions. (B) IG profiles with overnight (00:00-07:00 h) i.v. dextrose infusions at variable rates as indicated. (C) IG profiles at the time when the patient has been gradually weaned off the overnight i.v. dextrose infusions. Line in red indicates IG $<4 \mathrm{mmol} / \mathrm{L}$. * indicates capillary blood glucose measurement.

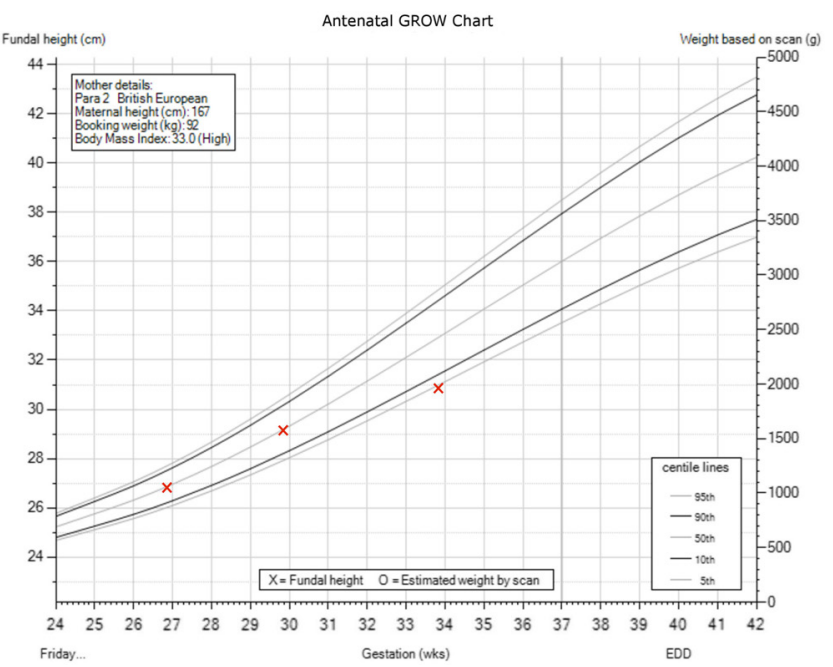

Figure 3

Antenatal weight chart. Gestational stage in week is on the $X$ axis, fundal height at the left $Y$ axis and estimated weight by scan on the right $Y$ axis.

\section{Outcome and follow-up}

The patient was readmitted under the care of the Obstetrics and Gynaecology team at 34/40 due to reduced fetal movements and an emergency caesarean section was indicated at $35+1 / 40$. She gave birth to a healthy baby girl with a birth weight of $2100 \mathrm{~g}$.

After delivery, the patient continued to monitor her BMs with finger prick testing, and her BM readings remained within the normal range with no further occurrence of hypoglycaemia. She was invited for a fasting plasma glucose test at 6 weeks postnatally, but did not attend to this or any further appointments.

\section{Discussion}

RYGB intervention is reported to result in DS in approximately $40 \%$ of patients, and it has previously been suggested that the presence of DS was essential for weight reduction, a hypothesis that has been refuted by further prospective studies (8). DS is characterised by vasomotor and GI symptoms caused by altered gastric emptying and subsequent rapid exposure of the small intestine to nutrients (1). Early DS is triggered by rapid passage of hyperosmolar content into the small bowel causing excessive release of GI hormones and shift of fluids into the GI lumen. It presents with abdominal pain, bloating, diarrhoea and vasomotor symptoms such as palpitations, flushing, sweating or tachycardia. Late DS is characterised by symptoms of hypoglycaemia and occurs typically between 1 and $3 \mathrm{~h}$ after meal ingestion (1). Rapid gastric emptying leads to expeditious glucose absorption 
from the small bowel leading to initial hyperglycaemia. Subsequent peak insulin secretion perpetuated by increased glucagon-like peptide 1 levels eventually causes hypoglycaemia $(1,9)$.

The advent of CGM technology enabled observation of abnormal glycaemic profiles in non-pregnant women after RYGB surgery. These are characterised by early, high and short-lasting IG peaks returning back to normal values before the usual time points for BM measurements after the $75 \mathrm{~g}$ OGTT ( 1 or $2 \mathrm{~h}$ post glucose challenge) (10). Interestingly, a subsequent study found very similar CGM profiles in pregnant women after RYGB (11). The peak IG value was reached at $54 \pm 9 \mathrm{~min}$ and the percentage of time spent with IG values above $140 \mathrm{mg} / \mathrm{dL}(7.8 \mathrm{mmol} / \mathrm{L})$ was similar to that observed in women with GDM (11). These findings indicate that the standard $75 \mathrm{~g}$ OGTT test is of poor diagnostic value of GDM in women post bariatric surgery. The other concern about the OGTT in this population is that the rapid absorption of dextrose can precipitate DS as discussed earlier.

Dietary modifications represent the mainstay of the management of late DS - patients are advised to avoid refined carbohydrates and increase the intake of high-GI food, which should be taken in multiple small portions throughout the day. Avoidance of drinks taken with food and recumbent position whilst eating are also recommended $(1,3)$. If dietary measures are not sufficient, use of acarbose, octreotide and diazoxide have been reported $(1,3)$. However, usage of these agents is limited by the lack of well-controlled studies examining their safety in pregnant women. In refractory cases, more invasive measures have been reported - continuous enteral feeding or a re-do surgery $(1,3)$. The patient from our report was aware of the paucity of safety data for these agents in pregnancy and refused to take any for this reason.

In our case, an emergency C-section at $35+1 / 40$ was indicated due to reduced fetal movements and a baby girl with a small-for-gestational-age birth weight of $2100 \mathrm{~g}$ was delivered. A large observational study from Sweden compared the outcomes of 670 pregnancies in women who had undergone bariatric surgery with control pregnancies matched for the mother's pre-surgery BMI, age, parity, smoking history, educational level and delivery year (12). Pregnancies after bariatric surgery were associated with higher risk for small-for-gestational-age infants and shorter gestation, but the risk of pre-term birth was not significantly different (12). The outcome of the pregnancy from our case therefore partially reflects these large cohort observations.
We cannot provide a compelling explanation as to why this patient's hypoglycaemic episodes started towards the end of the first trimester, neither can we explain why they stopped completely in the 34th week of her pregnancy, and she has remained asymptomatic until the present time. We can presume that pregnancy soon after the bariatric intervention contributed to the way how her symptoms presented. The pathophysiology of DS is not yet fully understood in the non-pregnant individuals either (1). We know from clinical observation that DS are common after pregnancy and can present a real diagnostic and therapeutic challenge (3). Normal pregnancy is a state of insulin resistance (IR) that gradually increases throughout its course with rising plasma insulin concentrations in an attempt to maintain normoglycaemia (13). We can therefore hypothesise that increased IR in later stages of pregnancy can trigger a higher post-prandial insulin secretion leading to subsequent hypoglycaemia (late dumping syndrome) in pregnant women with history of bariatric surgery, but further research will be required to confirm this hypothesis. Very recently, case reports of pregnant women with history of bariatric surgery suffering from dumping syndrome have been reported, and they seem to follow a similar time pattern with the occurrence of hypoglycaemic symptoms in 24th week of pregnancy in both cases $(14,15)$ and a nearly complete remission of symptoms after the delivery in one of the cases (15). Authors of the other case report do not comment on the presence or absence of symptoms after delivery (14). On the other hand, observational studies in pregnant women with type 1 diabetes show an increased risk of severe hypoglycaemia in 1st trimester and pregnancy-related intrinsic factors are postulated to play a role (16). Our patient did not experience any hypoglycaemic episodes during 1st trimester of her pregnancy, however.

Finally, the indications for the use of FL system in our patient were to avoid frequent nocturnal finger prick BM measurements that interfered with her sleep and as an educational tool with the aim to help her to identify hypoglycaemic triggers. The use FL had undoubtedly positive impact on the above, however, the ability to continuously check real-time IG levels led to a degree of anxiety and stress when IG readings were less than $4 \mathrm{mmol} / \mathrm{L}$ despite her being oligosymptomatic or asymptomatic on most occasions. The patient was very keen to receive inpatient care despite reassurances that she could safely be discharged. She had a complex social and psychological background, which caused additional distress during her admission. This led to a 
perceived dependence on midwifery staff. She used the data from FL as evidence that she was still having episodes of hypoglycaemia and leverage to extend her admission. We would therefore advise caution in CGM usage in individuals where excessive data may cause additional distress.

\section{Declaration of interest}

The authors declare that there is no conflict of interest that could be perceived as prejudicing the impartiality of the research reported.

\section{Funding}

This research did not receive any specific grant from any funding agency in the public, commercial or not-for-profit sector.

\section{Patient consent}

The authors confirm that written informed consent was obtained from the patient for publication of this article.

\section{Author contribution statement}

$P$ Novodvorsky attended to the patient and wrote the manuscript. E Walkinshaw, W Rahman, V Gordon, K Towse, S Mitchell, D Selvarajah, $\mathrm{P}$ Madhuvratha and $\mathrm{A}$ Munir attended to the patient and reviewed the manuscript.

\section{References}

1 Tack J \& Deloose E 2014 Complications of bariatric surgery: dumping syndrome, reflux and vitamin deficiencies. Best Practice and Research Clinical Gastroenterology 28 741-749. (https://doi.org/10.1016/j. bpg.2014.07.010)

2 Welbourn RSP, Finlay I, Sareela A, Somers S, Mahawar K, Walton P \& Kinsman R 2014 The United Kingdom National Bariatric Surgery Registry: Second Registry Report. Dendrite Clinical Systems. Published by the British Obesity \& Metabolic Society. Accesed from http://nbsr. co.uk/wp-content/uploads/2014/11/Extract_from_the_NBSR_2014_ Report.pdf

3 Narayanan RP \& Syed AA 2016 Pregnancy following bariatric surgery-medical complications and management. Obesity Surgery 26 2523-2529. (https://doi.org/10.1007/s11695-016-2294-x)
4 American Diabetes Association2017 Standards of medical care in Diabetes-2017. Diabetes Care 40 (Supplement 1) S4-S5. (https://doi. org/10.2337/dc17-S003)

5 Diabetes in pregnancy: management of diabetes and its complications from preconception to the postnatal period, 2015. (available at: https://www.nice.org.uk/guidance/ng3)

6 Adam S, Ammori B, Soran H \& Syed AA 2017 Pregnancy after bariatric surgery: screening for gestational diabetes. BMJ 356 j533. (https://doi.org/10.1136/bmj.j533)

7 Fokkert MJ, van Dijk PR, Edens MA, Abbes S, de Jong D, Slingerland RJ \& Bilo HJ 2017 Performance of the FreeStyle Libre Flash glucose monitoring system in patients with type 1 and 2 diabetes mellitus. BMJ Open Diabetes Research and Care 5 e000320. (https://doi.org/10.1136/bmjdrc-2016-000320)

8 Banerjee A, Ding Y, Mikami DJ \& Needleman BJ 2013 The role of dumping syndrome in weight loss after gastric bypass surgery. Surgical Endoscopy 27 1573-1578. (https://doi.org/10.1007/s00464012-2629-1)

9 Salehi M, Gastaldelli A \& D'Alessio DA 2014 Blockade of glucagonlike peptide 1 receptor corrects postprandial hypoglycemia after gastric bypass. Gastroenterology 146 669.e662-680.e662. (https://doi. org/10.1053/j.gastro.2013.11.044)

10 Hanaire H, Bertrand M, Guerci B, Anduze Y, Guillaume E \& Ritz P 2011 High glycemic variability assessed by continuous glucose monitoring after surgical treatment of obesity by gastric bypass. Diabetes Technology and Therapeutics 13 625-630. (https://doi. org/10.1089/dia.2010.0203)

11 Bonis C, Lorenzini F, Bertrand M, Parant O, Gourdy P, Vaurs C, Cazals L, Ritz P \& Hanaire H 2016 Glucose profiles in pregnant women after a gastric bypass : findings from continuous glucose monitoring. Obesity Surgery 26 2150-2155. (https://doi.org/10.1007/ s11695-016-2061-z)

12 Johansson K, Stephansson O \& Neovius M 2015 Outcomes of pregnancy after bariatric surgery. New England Journal of Medicine 372 2267. (https://doi.org/ 10.1056/NEJMc1503863)

13 Sonagra AD, Biradar SM, Dattatreya K \& Murthy DSJ 2014 Normal pregnancy - a state of insulin resistance. Journal of Clinical and Diagnostic Research 8 CC01-CC03. (https://doi.org/ 10.7860/ JCDR/2014/10068.5081)

14 Edawati DE, Abigail J, Mardiana K, Ming-Cheng C \& Harris NS 2017 Pregnancy and dumping syndrome post-bariatric surgery: a case report. Medical Journal of Malaysia 72 133-134.

15 Pacheco LV, Gilsanz CDP, Redondo LR, Navalón CI, Espín NVGT, Espína MBGT, Muñoz MR \& Avilés AM 2017 Late dumping syndrome in pregnant women after ROUX-EN-Y gastric bypass (RYGB) surgery. Presented at the 19th European Congress of Endocrinology, Lisbon, Portugal. Endocrine Abstracts 49 EP705. (https://doi.org/10.1530/ endocabs.49.EP705)

16 Evers IM, ter Braak EW, de Valk HW, van Der Schoot B, Janssen N \& Visser GH 2002 Risk indicators predictive for severe hypoglycemia during the first trimester of type 1 diabetic pregnancy. Diabetes Care 25 554-559. (https://doi.org/10.2337/diacare.25.3.554)

Received in final form 14 November 2017

Accepted 23 November 2017 